\title{
Review of: "The landscape of alternative polyadenylation in single cells of the developing mouse embryo"
}

\author{
Minmin Miao $^{1}$, Zhi-ping Zhang ${ }^{1}$, Hua BING
}

1 Yangzhou University

Potential competing interests: The author(s) declared that no potential competing interests exist.

This article is exciting and innovative. 3' untranslated regions (3' UTRs) regulate mRNA localization, degradation rates, and translational efficiency in post-transcriptional manner. The diversity of 3' -UTR isoforms is known as alternative polyadenylation (APA). As the importance of APA to mRNA function and mammalian development, the article generated a reliable poly (A) sites (PAS) set and links PASs to specific genes. In additional, this article evaluates global properties and gene-specific patterns of 3' -UTR shortening and lengthenin across mouse cell types and developmental time. Finally this article finds a large family of ELAV-like RBPs, putative regulators of alternative poly-adenylation, are induced in neuronal lineages and developmental stages experiencing 3 -UTR lengthening. This article sheds new light on the aspects of APAs in the developing mouse embryo. 